\title{
Trainees' Attitudes and Preferences towards the Use of Over the Counter Analgesics in Patients with Chronic Liver Disease
}

Nguyen D, Banerjee N, Abdelaziz D and Lewis $\mathrm{JH}^{*}$

Division of Gastroenterology, Hepatology Section, Department of Medicine, Georgetown University Medical Center, Washington, USA

\begin{abstract}
Background: The use of certain medications in patients with chronic liver disease and cirrhosis remains controversial. No formal evidence-based guidelines have been published regarding the use of acetaminophen or nonsteroidal anti-inflammatory drugs in patients in this setting. As a result, whether or not to prescribe these medications and at what dosages in patients with chronic liver disease or cirrhosis is often met with much consternation.

Objective: We assessed the prescribing preferences of senior medical students, internal medicine residents, and gastroenterology fellows for using NSAIDs and acetaminophen in patients with chronic liver disease (CLD), including those with cirrhosis.

Methods: A 21-question web-based survey was distributed to several major teaching hospitals in Washington, DC. An online survey software (Survey Monkey) was used to collect and analyze responses.

Results: A total of 543 trainees were sent the survey with $174(32 \%)$ responding. The majority of respondents who were willing to use acetaminophen recommended a daily dose of 2 gms or less regardless of their level of training. Internal medicine residents and senior medical students tended to recommend against acetaminophen at any dose in favor of NSAIDs in decompensated cirrhotics. All trainee levels showed a diminishing preference towards using a therapeutic dose of $4 \mathrm{gms}$ acetaminophen per day as a function of CLD severity.

Conclusions: There is a wide divide at the trainee level regarding the usage and dosing for acetaminophen in patients with chronic liver disease. Additional education on the safe use of NSAIDs and acetaminophen in CLD and cirrhosis needs to begin in medical school. Senior students in particular voiced the need for controlled prospective studies in order to develop evidence-based guidelines to determine the appropriate indications for use of NSAIDs and acetaminophen along the spectrum of hepatic impairment in chronic liver disease.
\end{abstract}

Keywords: Cirrhosis; Physician prescribing patterns; Acetaminophen; Academic training; NSAIDS

\section{Introduction}

\section{Objective}

The safe use of medications in patients with Chronic Liver Disease (CLD) and cirrhosis remains clinically challenging [1]. Increasing degrees of hepatic impairment may alter the disposition and effects of many drugs through changes in pharmacokinetics and end-organ response. The greater the degree of liver dysfunction, the higher the potential for impaired drug metabolism with the risk of adverse hepatic and other drug effects [1-3]. This may be especially true when it comes to the use of OTC prescription analgesics.

Intentional Acetaminophen (APAP) overdose is the most common cause of acute liver failure in the United States [4], and as a result, the drug is often perceived by many patients and clinicians alike as being too dangerous to use in patients with any form of chronic liver disease, especially cirrhosis, even when therapeutic doses are taken [1,5-7]. While a number of authors have suggested that 2-3 gm/day of APAP given for short durations is safe in patients with cirrhosis [1,5,7-9], this recommendation is largely based on anecdotal clinical experience and expert opinion. As a result, many clinicians still suggest that their chronic liver disease patients, especially those with cirrhosis, not use APAP in any dose or under any circumstances $[1,10]$.

Nonsteroidal Anti-Inflammatory Drugs (NSAIDs) as a group carry a class warning about the possibility of hepatic injury, raising the possibility of acute on chronic injury developing in a cirrhotic patient
[11]. However, a more likely concern with NSAID use in cirrhosis is renal impairment [12], in particular precipitating hepatorenal syndrome - a frequently fatal complication of advanced liver disease [13]. Therefore, it is usually recommended that NSAIDs be avoided in cirrhosis, especially those with ascites [14]. NSAIDs can also cause mucosal bleeding or worsen acute bleeding in patients at increased risk of bleeding as a result of the thrombocytopenia and coagulopathy associated with advanced liver disease $[1,15,16]$. This risk may even be greater in patients with portal hypertension-related complications, such as esophageal or gastric varices, portal hypertensive gastropathy, or Gastric Antral Vacular Ectasias (GAVE) [15,17].

The work of a limited number of investigators has helped to clarify the usage patterns of Over the Counter Analgesics (OTCAs) including APAP and various NSAIDs with respect to their safety in cirrhosis. Khalid et al. [18] found that among non-cirrhotic patients

*Corresponding author: Lewis JH, Division of Gastroenterology, Hepatology Section Department of Medicine, Georgetown University Medical Center, Washington, USA, Tel: 202-444-8571, Fax: 202-444-4211; E-mail: lewisjh@gunet.georgetown.edu

Received January 23, 2014; Accepted October 27, 2014; Published October 28 2014

Citation: Nguyen D, Banerjee N, Abdelaziz D, Lewis JH (2014) Trainees' Attitudes and Preferences towards the Use of Over the Counter Analgesics in Patients with Chronic Liver Disease. Adv Pharmacoepidemiol Drug Saf 3: 167 doi:10.4172/2167-1052.1000167

Copyright: ( 2014 Lewis JH, et al. This is an open-access article distributed under the terms of the Creative Commons Attribution License, which permits unrestricted use, distribution, and reproduction in any medium, provided the original author and source are credited. 
in their subspecialty liver clinic, 70\% used APAP or NSAIDs. In their patients with compensated cirrhosis, over half mentioned the use of over the counter analgesics, with 25\% taking APAP and 31\% receiving NSAIDs. Among those with advanced cirrhosis who were hospitalized for a number of common causes of hepatic decompensation, a lower percentage (35\%) mentioned taking over the counter analgesics with $19 \%$ saying they used acetaminophen and $16 \%$ took NSAIDs [14]. Of importance was the fact that hospitalization rates were not increased among cirrhotics using these over the counter analgesics, as reported by Fenkel et al. [19].

In 2008, Rossi et al. studied physicians' attitudes regarding the use of over-the-counter nonprescription analgesics in CLD [10]. The results of their web-based questionnaire survey found that internists and family physicians were significantly more likely not to recommend the use of acetaminophen in patients with compensated cirrhosis compared to gastroenterologists, who felt that APAP would, in fact, be safe. In patients with decompensated cirrhosis, $95 \%$ of family physicians and $70 \%$ of internists would not recommend the use of acetaminophen compared to just $22 \%$ of gastroenterologists. Even among patients with mild chronic hepatitis without cirrhosis, 15 to $20 \%$ of general practitioners would not recommend APAP. In contrast, none of the gastroenterologists questioned in their survey would avoid APAP in that setting. Overall, non-gastroenterologists were more likely to recommend NSAIDs compared to gastroenterologists who were more likely to recommend the use of APAP [10]. Their survey, however, did not address the specific reasoning behind these recommendations, nor did it specify what daily APAP dose the physicians responding would recommend.

It is unclear whether or not medical students or physicians in training (medical residents or GI fellows) are aware of, or put any of these findings to use. We are also unaware of any information regarding trainees' reasoning for or against prescribing OTCAs in CLD patients, nor their familiarity with any APAP dosing recommendations. This study was designed to examine the preferences and perspectives of senior medical students (MS4), internal medicine residents (PGY I,II,III), and gastroenterology fellows, in order to identify their attitudes and prescribing habits for NSAIDs and APAP patients with CLD and cirrhosis.

\section{Design}

A 21-question web-based survey was designed and distributed via email. Included trainees were senior medical students, residents and GI fellows at several of the major teaching institutions in Washington, DC. These included Medstar Georgetown Hospital, Medstar Washington Hospital Center, and George Washington University Hospital. There were no specific exclusion criteria; the survey was not sent to anybody outside of the aforementioned training designations. The survey sought to determine what recommendations they would make regarding NSAID and APAP use in patients with chronic hepatitis without cirrhosis of any cause (group I), in compensated cirrhosis (group II), and in decompensated cirrhosis (group III). During 2012, the email was sent 6 separate times to maximize responses and all results were kept confidential. An online survey software (Survey Monkey) was used to collect and analyze responses. The questionnaire can be found in the supplemental section as an attached PDF.

The study was submitted to and approved by the Georgetown Institutional Review Board prior to online distribution of the survey. No statistical analysis software was used.

\section{Results}

The survey was sent to a total of 543 trainees. A total of 177 responses were collected, accounting for a $33 \%$ overall response rate. The survey was answered by 72 senior medical students, 86 internal medicine residents (35 PGY-1, 23 PGY-2, 28 PGY-3), and 19 GI fellows (in all 3 years of training).

The respondents' preferences and recommendations for APAP and NSAID use based on the severity of the CLD and their level of training are summarized in Table 1. Overall, the willingness to use APAP decreased as liver disease severity increased; $62 \%$ of MS4s would recommend APAP use in chronic hepatitis patients, but this decreased to only $4 \%$ in decompensated cirrhotics. Similar trends were observed for the PGY1-3 medicine residents regarding recommendations for APAP use from CLD group I to III, from $74 \%$ to $19 \%$ in PGY1s, $57 \%$ to $14 \%$ in PGY2s and from $89 \%$ to $4 \%$ in PGY3s. GI fellows were the only group of trainees in which the majority continued to favor APAP use for every stage of liver disease, with only a small drop from $89 \%$ to $79 \%$ in the recommendation as CLD severity progressed from groups I to III. In comparison, this trend was reversed with NSAID use. Only $5 \%$ of GI fellows recommended NSAID use for any stage of CLD. In contrast, among the other trainees (medical students and internal medicine residents), the majority recommended NSAID usage in groups I and II. MS4s were most likely to recommend NSAID use for any stage of liver disease, with $96 \%$ recommending use in groups I and II and 58\% recommending NSAID use in group III.

Trainees were also surveyed regarding the maximum daily APAP dose that they would recommend based on the severity of liver disease, with the results summarized in Table 2. The maximum therapeutic dose of $4 \mathrm{gm}$ daily of APAP was recommended least often for any stage of chronic liver disease at every level of physician training. In contrast, a maximum daily dose of $2 \mathrm{gm}$ APAP was most often recommended for any stage of chronic liver disease, although responses were not unanimous amongst the trainees. Those who most strongly favored the 2 gm maximum daily dose were the MS4s and GI fellows, with over $60 \%$ expressing that dose preference for any stage of CLD. PGY 1-3s were more hesitant about the safety of any daily dose, with a majority feeling that $2 \mathrm{gm}$ per day of APAP was acceptable for groups I and II while no maximal daily dose was acceptable for group III.

Medical students and internal medicine and GI physician trainees were also surveyed as to why they would avoid NSAIDs and/or APAP in patients with CLD. As Table 3 shows, there was wide variability in the reasons cited to avoid NSAID use in this setting. One-third of MS4s, $44 \%$ of PGY1s, $80 \%$ of PGY2s, $25 \%$ of PGY3s, and $22 \%$ of GI fellows expressed concern that NSAIDs would increase the risk of gastric ulcers and/or would precipitate GI bleeding. The risk of renal

\begin{tabular}{|c|c|c|c|c|c|c|c|c|c|c|}
\hline & \multicolumn{2}{|c|}{ MS4 N=72 } & \multicolumn{2}{|c|}{ PGY1 N=35 } & \multicolumn{2}{|c|}{ PGY2 N=23 } & \multicolumn{2}{|c|}{ PGY3 N= 28} & \multicolumn{2}{|c|}{ GI Fellow $\mathbf{N}=19$} \\
\hline & APAP & NSAID & APAP & NSAID & APAP & NSAID & APAP & NSAID & APAP & NSAID \\
\hline Group I & $62 \%$ & $96 \%$ & $74 \%$ & $61 \%$ & $57 \%$ & $71 \%$ & $89 \%$ & $85 \%$ & $89 \%$ & $5 \%$ \\
\hline Group II & $32 \%$ & $96 \%$ & $55 \%$ & $56 \%$ & $61 \%$ & $57 \%$ & $59 \%$ & $69 \%$ & $89 \%$ & $5 \%$ \\
\hline Group III & $4 \%$ & $58 \%$ & $19 \%$ & $25 \%$ & $13 \%$ & $29 \%$ & $4 \%$ & $27 \%$ & $79 \%$ & $5 \%$ \\
\hline
\end{tabular}

Table 1: Percentage of trainees by level of training who recommended APAP or NSAIDs (dose not specified) at varying levels of CLD. 


\begin{tabular}{|c|c|c|c|c|c|}
\hline & \multicolumn{5}{|c|}{4 gms daily } \\
\hline & MS4 N=72 & PGY1 N=35 & PGY2 N=23 & PGY3 N=28 & GI Fellow $\mathrm{N}=19$ \\
\hline Group I & $22 \%$ & $23 \%$ & $22 \%$ & $22 \%$ & $26 \%$ \\
\hline Group II & $8 \%$ & $3 \%$ & $9 \%$ & $8 \%$ & $0 \%$ \\
\hline \multirow[t]{3}{*}{ Group III } & $0 \%$ & $0 \%$ & $0 \%$ & $4 \%$ & $0 \%$ \\
\hline & \multicolumn{5}{|c|}{2 gms or less daily } \\
\hline & MS4 N=72 & PGY1 N=35 & PGY2 N=23 & PGY3 N=28 & GI Fellow $\mathrm{N}=19$ \\
\hline Group I & $61 \%$ & $45 \%$ & $26 \%$ & $48 \%$ & $63 \%$ \\
\hline Group II & $62 \%$ & $43 \%$ & $52 \%$ & $36 \%$ & $84 \%$ \\
\hline \multirow[t]{3}{*}{ Group III } & $63 \%$ & $24 \%$ & $17 \%$ & $4 \%$ & $73 \%$ \\
\hline & \multicolumn{5}{|c|}{ No daily dose is acceptable } \\
\hline & MS4 $\mathrm{N}=72$ & PGY1 N=35 & PGY2 N=23 & PGY3 N=28 & GI Fellow $\mathrm{N}=19$ \\
\hline Group I & $6 \%$ & $26 \%$ & $43 \%$ & $11 \%$ & $11 \%$ \\
\hline Group II & $15 \%$ & $47 \%$ & $39 \%$ & $32 \%$ & $11 \%$ \\
\hline Group III & $36 \%$ & $76 \%$ & $83 \%$ & $92 \%$ & $21 \%$ \\
\hline
\end{tabular}

Table 2: Percentage of trainees who recommended a specified daily dose of APAP at given stages of CLD.

\begin{tabular}{|l|c|c|c|c|c|}
\hline & $\begin{array}{c}\text { MS4 } \\
\text { N=72 }\end{array}$ & $\begin{array}{c}\text { PGY-1 } \\
\text { N=35 }\end{array}$ & $\begin{array}{c}\text { PGY-2 } \\
\text { N=23 }\end{array}$ & $\begin{array}{c}\text { PGY-3 } \\
\text { N=28 }\end{array}$ & $\begin{array}{c}\text { GI Fellow } \\
\text { N=19 }\end{array}$ \\
\hline $\begin{array}{l}\text { Risk of precipitating } \\
\text { ulcers/GI bleeding }\end{array}$ & $33 \%$ & $44 \%$ & $80 \%$ & $25 \%$ & $22 \%$ \\
\hline Risk of renal failure & $24 \%$ & $33 \%$ & $20 \%$ & $25 \%$ & $67 \%$ \\
\hline $\begin{array}{l}\text { Concern about worsening } \\
\text { underlying liver disease }\end{array}$ & $20 \%$ & $11 \%$ & NS & $50 \%$ & $11 \%$ \\
\hline $\begin{array}{l}\text { Preference for APAP over } \\
\text { NSAIDS }\end{array}$ & $23 \%$ & $12 \%$ & NS & NS & NS \\
\hline
\end{tabular}

Table 3: Primary reasons given for not wanting to use NSAIDs in patients with CLD.

\begin{tabular}{|l|c|c|c|c|c|}
\hline & $\begin{array}{c}\text { MS4 } \\
\text { N=72 }\end{array}$ & $\begin{array}{c}\text { PGY-1 } \\
\text { N=35 }\end{array}$ & $\begin{array}{c}\text { PGY-2 } \\
\text { N=23 }\end{array}$ & $\begin{array}{c}\text { PGY-3 } \\
\text { N=28 }\end{array}$ & $\begin{array}{c}\text { GI Fellow } \\
\text { N=19 }\end{array}$ \\
\hline $\begin{array}{l}\text { Concern of worsening } \\
\text { underlying liver disease }\end{array}$ & $33 \%$ & $33 \%$ & $100 \%$ & $100 \%$ & $100 \%$ \\
\hline $\begin{array}{l}\text { Concern of inadvertent } \\
\text { overdose }\end{array}$ & $31 \%$ & $67 \%$ & NS & NS & NS \\
\hline $\begin{array}{l}\text { Lack of information regarding } \\
\text { use of APAP in CLD patients }\end{array}$ & $36 \%$ & NS & NS & NS & NS \\
\hline
\end{tabular}

Table 4: Primary reasons for not wanting to use of APAP in patients with CLD.

failure with NSAID use was the primary concern of $24 \%$ of MS4s, $33 \%$ of PGY1s, $20 \%$ of PGY2s, $25 \%$ of PGY3s, and $67 \%$ of GI fellows. Twenty percent of MS4s, $11 \%$ of PGY1s, $0 \%$ of PGY2s, $50 \%$ of PGY3s, and $11 \%$ of GI fellows felt that worsening of the underlying liver disease was the primary reason to avoid NSAIDs.

Specific reasons why trainees would not use APAP in CLD patients are shown in table 4 . All of the PGY- 2 and 3 residents and GI fellows who did not recommend use of APAP in CLD patients were primarily concerned over worsening of underlying liver disease. Two thirds of PGY1 residents were concerned with the risk of an inadvertent overdose. MS4s were more split in their reasoning between a risk of worsening of the underlying disease, concern about an inadvertent overdose and the lack of formal guidelines on APAP use in CLD patients. No trainee groups avoided APAP due to a preference for using NSAIDs or narcotics.

\section{Discussion}

The results of this survey show that for every stage of CLD, senior level medical students favored the use of NSAIDs over APAP. Internal medicine residents also preferred NSAIDs over APAP in nearly every stage of CLD. In contrast, GI fellows overwhelmingly favored the use of APAP over NSAIDs at every stage of CLD. We did note, however, that the willingness to prescribe either type of OTCA decreased as a function of CLD severity in most trainee groups. These findings among medical students and internal medicine residents are very similar to previously published studies [10]. The reasons given by the trainees, who were worried about using APAP, primarily reflected their concerns about worsening the underlying liver disease, precipitating an inadvertent overdose, and the lack of evidence-based information on which to justify clinical decisions.

Our study indicates that a significant variability exists among health care providers and physicians in training regarding their recommendations on the use of OTCAs, and affords an opportunity for physician and patient education on medication use in CLD. Nonspecialty trainees (senior medical students and IM residents) were much more willing to use NSAIDs at all levels of CLD as compared with GI fellows- a trend not seen between general practitioners and GI specialists. However, the trend towards avoiding NSAID use in Group III cirrhotics held is similar to results from Rossi et al. [10].

The recommendations for using APAP differed significantly among practicing physicians in the study by Rossi et al for all levels of CLD severity [10]. APAP was deemed safe for use by $100 \%, 95 \%$, and $80 \%$ of gastroenterologists for patients in groups I, II, III respectively. In contrast, APAP use was deemed suitable for CLD groups I, II, III by $85-90 \%, 50-80 \%$, and $10-30 \%$ of general internists (GIMs) and family practitioners (FPs) respectively. The gastroenterologists' recommendations for APAP use were similar to those of the GI fellows in our survey for all levels of CLD. While the recommendations for APAP use varied among senior level medical students and residents and among practicing FPs/GIMs, the overall trend of using APAP less in patients with more advanced stages of CLD (group III) was confirmed in both studies. GI fellows and practicing gastroenterologists were both more likely to recommend APAP over NSAIDs at the most advanced stages of CLD (groups II, and III).

Although no formal evidence-based guidelines have been published regarding the use of APAP or NSAIDs in patients with CLD or cirrhosis, expert opinion suggests that a daily dose of 2-3gm or less of APAP is safe for short term use in patients with CLD including cirrhosis[1,5-10]. While APAP remains the single leading cause of acute liver failure in the US, the UK and other westernized countries, the majority of instances involve an intentional overdose $[4,20]$. In contrast, the vast majority of APAP users take the drug safely and without incident [21]. Although it has been demonstrated that doses of 4 grams daily over the course of 2 weeks given to healthy volunteers can lead to marked, but clinically silent, elevations in ALT and AST in more than $40 \%$ of subjects [22], other groups have not shown significant ALT elevations with doses up to 8 grams daily when used for shorter periods $[23,24]$, including in patients who have recently used alcohol [25]. The issue of "therapeutic misadventure", a termed coined by Maddrey and Zimmerman in the mid-1990s [26], is still a cautionary tale, in that unsuspecting chronic alcohol users (which can include cirrhotics), may experience an inadvertent overdose from APAP (in doses far lower than the traditional 10 grams implicated in most intentional overdoses) leading to acute on chronic liver failure. However, such reports remain relatively anecdotal and limited in number compared to the billions of doses taken annually [21,27], and no instances of acute liver failure were seen with daily doses less than $2.5 \mathrm{gm}$ [26]. Moreover, the toxic-metabolic premises upon which the alcohol-acetaminophen interaction was based (i.e. increased formation of NAPQI by CYP2E1 induction, and glutathione depletion by alcohol), have been challenged $[25,28]$. Unfortunately, there are no long-term prospective studies looking at APAP use in cirrhotics. 
In contrast to the concerns about using APAP in patients with CLD, most lay persons and many physicians consider NSAIDs to be a safer analgesic option. However, owing to their inhibition of prostaglandin synthesis, NSAIDs are generally not recommended for use in cirrhosis due to the risk of precipitating GI bleeding and renal failure [1,12-17]. Patients with cirrhosis have increased synthesis of renal prostaglandins to counteract the renin-angiotensin-aldosterone and sympathetic systems that reduce perfusion to the kidneys [14]. Circulating renal prostaglandins help to maintain and regulate renal hemodynamics, glomerular filtration and the renal handling of sodium and water. In addition, NSAIDs are largely protein bound and metabolized by CYP P450 enzymes and thus increased serum levels can be anticipated in cirrhosis [29]. The inhibition of prostaglandin synthesis leads to a profound decrease in renal perfusion, reduction in GFR, impairment in water clearance, and marked sodium retention. Moreover, NSAIDs have deleterious effects on platelets that can increase the risk of GI hemorrhage [30]. In addition, ibuprofen has been associated with exacerbating chronic hepatitis $C$, suggesting it might not be safe in this setting [31], although others have challenged this notion [32]. Although celecoxib may not impair platelet or renal function in cirrhosis to the extent of non-selective NSAIDs, its long-term safety in cirrhosis remains to be established [33]

In general, the majority of physician trainees in our survey believed that a daily APAP dose of 2 gm or less was safe for some CLD patients, regardless of their level of training. However, for patients with decompensated cirrhosis, a majority of IM residents felt that no daily dose of APAP was safe. Moreover, all trainee levels showed a diminished preference for a full dose of $4 \mathrm{gms}$ per day as a function of CLD severity. When offered a range of APAP doses, most trainees in our survey had the strongest preference for using a daily dose of $2 \mathrm{gms}$ or less in patients with chronic hepatitis or stable cirrhosis. For decompensated cirrhosis, the majority of trainees felt that no daily dose was acceptable.

In 2011, in an effort to help encourage and ensure appropriate acetaminophen use, the maker of Extra Strength TYLENOL implemented new dosing instructions that lowered the maximum daily dose for single-ingredient Extra Strength TYLENOL products sold in the U.S. from 8 pills per day $(4,000 \mathrm{mg})$ to 6 pills per day $(3,000$ $\mathrm{mg}$ ). The dosing interval has also changed from 2 pills every $4-6$ hours to 2 pills every 6 hours [34]. Although these recommendations were for the general population at large, with no specific guidelines offered for patients with CLD, there is evidence to suggest that APAP taken at therapeutic doses may be safe in patients with CLD. Fenkel et al. demonstrated that "non-excessive" doses of OTCAs (NSAIDs and/or APAP) in patients with cirrhosis were not associated with an increased rate of hospitalization for liver related events [19]. The non-excessive dosage referred to in the study were listed as being less than the maximally recommended dose in the package label, although actual dosages in the study were not reported. Khalid et al found that reduced doses of APAP were not associated with acute hepatic decomposition, even among patients with recent alcohol ingestion [18].

In examining the reasons for why trainees would avoid using NSAIDs or APAP in CLD patients, their responses were found to be variable. The majority of MS4s and IM residents were concerned about NSAIDs causing ulcers or GI bleeding in patients with CLD, whereas GI fellows were more concerned about NSAIDs precipitating renal failure in these patients. The study by Rossi et al. [10] did not address the specific reasoning behind the practitioners' recommendations for or against NSAID use in CLD patients, and thus no direct comparison can be made with our respondents. However, most senior level medical residents and GI fellows were concerned that APAP could worsen the underlying liver disease. Of note, senior medical students were the only group to suggest that the lack of evidence-based literature on the subject was the main reasoning behind their avoidance of APAP.

An interesting observation highlighted by the results of our survey, and also found by Rossi et al. [10], is why a preference for NSAIDs over APAP in CLD patients still exists. Given that NSAID-induced GI and renal toxicity and idiosyncratic hepatotoxicity have been well characterized in the literature, coupled with the evidence that APAP at low or therapeutic doses is generally safe in CLD patients, some might find it surprising that both primary care practitioners and nonGI trainees consistently chose NSAIDs over APAP at all levels of CLD severity. Based on the responses provided by our survey, it seems that while senior medical students and internal medicine residents are aware of the GI toxicity of NSAIDs, they appear to be less concerned with the risk of renal failure compared to GI fellows. It is possible that the often dramatic events surrounding acute liver failure associated with APAP outweighs the concern for NSAID-associated GI toxicity among nonGI physicians. While one might surmise that the absolute risk of renal failure might be a stronger reason to change their preference, at what level of training and how this information should this is introduced has not been studied. Nevertheless, it appears that given the responses to our survey, both trainees and general medical practitioners have an incomplete understanding of the potential for NSAID and APAP to cause toxicity in CLD patients.

Regarding information given to patients about using OTCAs, only about half of the patients included in the survey by Fenkel et al. ever reported having received medical advice on OTCA use by pharmacists or physicians [19]. The reluctance of trainees responding to our survey to use APAP at any dose in decompensated cirrhosis implies that further evidenced-based recommendations are needed for this patient population. Longer-term prospective studies examining the safety of APAP in CLD patients would bolster and extend recommendations that are currently based largely on expert opinion.

\section{Conclusion}

Our results show that there exists a divide, even at the training level, among IM residents and those specifically training to become gastroenterologists with respect to selecting OTCAs for use in patients with CLD. We also observed a preference for recommending that APAP be used in lieu of NSAIDs in CLD patients of any stage among trainees in GI fellowships. Senior medical students and internal medicine residents were more likely to recommend NSAIDs over APAP for CLD patients, with a majority still preferring NSAID use, even in patients with decompensated cirrhosis. Our survey results also found a variable response to what trainees consider to be the maximum daily safe dose of APAP for the various stages of CLD and the reasons why some trainees felt that NSAIDs should be avoided. Although $2 \mathrm{gm}$ per day of APAP was the most agreed upon dose, this is an indication that additional education is needed beginning in medical school. Prospective studies would be welcomed to help determine a safe dose of APAP and duration of treatment in CLD patients.

\section{References}

1. Lewis JH, Stine JG (2013) Review article: prescribing medications in patients with cirrhosis-a practical guide. Aliment Pharmacol Ther 37: 1132-1156.

2. Delcò F, Tchambaz L, Schlienger R, Drewe J, Krähenbühl S (2005) Dose adjustment in patients with liver disease. Drug Saf 28: 529-545. 
Citation: Nguyen D, Banerjee N, Abdelaziz D, Lewis JH (2014) Trainees' Attitudes and Preferences towards the Use of Over the Counter Analgesics in Patients with Chronic Liver Disease. Adv Pharmacoepidemiol Drug Saf 3: 167. doi:10.4172/2167-1052.1000167

Page 5 of 5

3. Verbeeck RK (2008) Pharmacokinetics and dosage adjustment in patients with hepatic dysfunction. Eur J Clin Pharmacol 64: 1147-1161.

4. Lee WM (2013) Drug-induced acute liver failure. Clin Liver Dis 17: 575-586.

5. Benson GD, Koff RS, Tolman KG (2005) The therapeutic use of acetaminophen in patients with liver disease. Am J Ther 12: 133-141.

6. Murphy EJ (2005) Acute pain management pharmacology for the patient with concurrent renal or hepatic disease. Anesthesia Intensive Care 33: 311-322.

7. Gupta NK, Lewis JH (2008) Review article:The use of potentially hepatotoxic drugs in patients with liver disease. Aliment Pharmacol Ther 28: 1021-1041.

8. Chandok N, Watt KD (2010) Pain management in the cirrhotic patient: the clinical challenge. Mayo Clin Proc 85: 451-458.

9. Lucena MI, Andrade RJ, Tognoni G, Hidalgo R, Sanchez de la Cuesta F, et al. (2003) Drug use for non-hepatic associated conditions in patients with liver cirrhosis. Eur J Clin Pharmacol 59: 71-76.

10. Rossi S, Assis DN, Awsare M, Brunner M, Skole K, et al. (2008) Use of overthe-counter analgesics in patients with chronic liver disease: physicians' recommendations. Drug Saf 31: 261-270.

11. Lewis JH (1998) NSAID-induced hepatotoxicity. Clin Liver Dis 2: 543-561.

12. Brater DC, Anderson SA, Brown-Cartwright D, Toto RD (1986) Effects of nonsteroidal antiinflammatory drugs on renal function in patients with renal insufficiency and in cirrhotics. Am J Kidney Dis 8: 351-355.

13. Salerno F, Gerbes A, Gines P, Wong F, Arroyo V (2007) Diagnosis, prevention and treatment of hepatorenal syndrome in cirrhosis. Gut 56: 1310-1318.

14. Laffi G, La Villa G, Pinzani M, Marra F, Gentilini P (1997) Arachidonic acid derivatives and renal function in liver cirrhosis. Semin Nephrol 17: 530-548.

15. Lee YC, Chang CH, Lin JW, Chen HC, Lin MS, et al. (2012) Non-steroidal antiinflammatory drugs use and risk of upper gastrointestinal adverse events in cirrhotic patients. Liver Int 32: 859-866.

16. Castro-Fernandez M, Sanchez-Munoz D, Galan-Jurado MV, Larraona JL, Suárez E, et al. (2006) Influence of nonsteroidal antiinflammatory drugs in gastrointestinal bleeding due to gastroduodenal ulcers or erosions in patients with liver cirrhosis. Gastroenterol Hepatol 29: 11-14.

17. Deledinghen V, Heresbach D, Fourdan O, Bernard P, Liebaert-Bories M, et al. (1999) Anti-inflammatory drugs and variceal bleeding: a case-control study. Gut 44: 270-273.

18. Khalid SK, Lane J, Navarro V, Garcia-Tsao G (2009) Use of over-the-counter analgesics is not associated with acute decompensation in patients with cirrhosis. Clin Gastroenterol and Hepatol 7: 994-999.

19. Fenkel JM, Coron RN, Daskalakis C, Vega M, Rossi S, et al. (2010) Overthe-counter analgesics in cirrhotic patients: a case-control study examining the risk of hospitalization for liver-associated events. Scand J Gastroenterol 45 1101-1109.

20. Prescott LF (2000) Therapeutic misadventure with paracetamol: fact or fiction? Am J Ther 7: 99-114.

21. Jones A (2002) Over-the-counter analgesics: a toxicology perspective. Am J Ther 9: 245-257.

22. Watkins PB, Kaplowitz N, Slattery JT, Colonese CR, Colucci SV, et al. (2006) Aminotransferase elevations in healthy adults receiving 4 grams of acetaminophen daily: a randomized controlled trial. JAMA 296: 87-93.

23. Temple AR, Lynch JM, Vena J, Auiler JF, Gelotte CK, et al. (2007) Aminotransferase activities in healthy subjects receiving three-day dosing of 4 6, or 8 grams per day of acetaminophen. Clin Toxicol (Phila) 45: 36-44.

24. Heard KJ, Green JL, Dart RC (2010) Serum alanine aminotransferase elevation during 10 days of acetaminophen use in nondrinkers. Pharmacotherapy 30 818-822.

25. Rumack B, Heard K, Green J, Albert D, Bartelson BB, et al. (2012) The effect of acetaminophen on serum alanine aminotransferase activity in subjects who consume ethanol: a systematic review and meta-analysis of published randomized, controlled trials. Pharmacotherapy 32: 784-91.

26. Zimmerman HJ, Maddrey WC (1995) Acetaminophen (paracetamol) hepatotoxicity with regular intake of alcohol: analysis of instances of therapeutic misadventure. Hepatology 22: 767-773.

27. Dart RC, Green JL, Kuffner EK, Heard K, Sproule B, et al. (2010) The effects of paracetamol (acetaminophen) on hepatic tests in patients who chronically abuse alcohol-a randomized study. Aliment Pharmacol Ther 32: 478-486.

28. Rumack BH (2004) Acetaminophen misconceptions. Hepatology 40:10-15

29. Bosilkovska M, Walder B, Besson M, Daali Y, Desmeules J (2012) Analgesics in patients with hepatic impairment: pharmacology and clinical implications. Drugs 72:1645-1669.

30. Schafer Al (1995) Effects of nonsteroidal antiinflammatory drugs on platelet function and systemic hemostasis. J Clin Pharmacol 35: 209-219.

31. Riley TR $3^{\text {rd }}$, Smith JP (1998) Ibuprofen-induced hepatotoxicity in patients with chronic hepatitis C: a case series. Am J Gastroenterol 93: 1563-1565.

32. Andrade RJ, Lucena MI, Garcia-Cortes M, García-Ruiz E, Fernández-Bonilla $\mathrm{E}$, et al. (2002) Chronic hepatitis C, ibuprofen, and liver damage. Am J Gastroenterol 97: 1854-1855.

33. Claria J, Kent JD, Lopez-Parra M, Escolar G, Ruiz-Del-Arbol L, et al. (2005) Effects of celecoxib and naproxen on renal function in nonazotemic patients with cirrhosis and ascites. Hepatology 41: 579-587.

34. McNEIL-PPC (2013) Acetaminophen Dosage for Adults. 